\title{
Manejo médico y quirúrgico de los efectos adversos asociados al uso de filtros de vena cava inferior
}

\section{Medical and surgical management of adverse effects associated with inferior vena cava filters}

\author{
Luis A. Rojas-Trejo, Guillermo Castro-Lozano, Elena del C. Pacheco-Perea y Adriana Torres-Machorro* \\ Departamento de Cirugía, Instituto Nacional de Cardiología Ignacio Chávez, Ciudad de México, México
}

\begin{abstract}
Resumen
Los filtros de vena cava se desarrollaron con el objetivo de prevenir la embolia pulmonar y reducir la mortalidad asociada a tromboembolia venosa. Su utilización es variable de acuerdo con el tipo de guía consultada. El implante de estos filtros puede llevar a complicaciones, especialmente si no se realiza un seguimiento adecuado. Dentro de las complicaciones con mayor frecuencia en la colocación de filtros de vena cava se encuentran dificultades en el acceso, mala posición, expansión incompleta con migración, trombosis, fractura del filtro, perforación de las paredes de la vena cava e infección del dispositivo. El retiro de estos dispositivos requiere de un tiempo quirúrgico prolongado y la utilización de técnicas no convencionales. Presentamos el caso de una paciente de 43 años con síndrome de anticuerpos antifosfolípidos, múltiples episodios de trombosis venosa y embolia pulmonar, portadora de un filtro en vena cava inferior que presenta trombosis y perforación de las paredes de la vena cava.
\end{abstract}

Palabras clave: Trombosis de filtro de vena cava. Perforación de vena cava inferior. Trombosis venosa profunda. Embolia pulmonar.

\begin{abstract}
Vena cava filters were developed to prevent pulmonary embolism and reduce mortality associated with venous thromboembolism. Its use varies according to the guidelines that are reviewed, and the implantation of these filters may lead to complications; especially if there is no follow-up. Among the complications due to the placement of vena cava filters, are access complications, poor position of the filter, incomplete expansion with migration, thrombosis, filter fracture, perforation of the vena cava and device infection. The removal of these devices requires a prolonged surgical time and the use of unconventional techniques. We present the case of a 43-year-old patient with antiphospholipid antibody syndrome, multiple episodes of venous thrombosis and pulmonary embolism, who had an inferior vena cava filter and presented thrombosis and perforation.
\end{abstract}

Keywords: Vena cava filter thrombosis. Inferior vena cava perforation. Pulmonary embolism. Deep vein thrombosis.

Correspondencia:

*Adriana Torres-Machorro

E-mail: atorres.machorro@gmail.com
Disponible en internet: 23-02-2022

Rev Mex Angiol. 2022;50(1):32-39 www.RMAngiologia.com 0377-4740/@ 2021 Sociedad Mexicana de Angiología y Cirugía Vascular y Endovascular, A.C. Publicado por Permanyer. Este es un artículo open access bajo la licencia CC BY-NC-ND (http://creativecommons.org/licenses/by-nc-nd/4.0/). 


\section{Introducción}

La anticoagulación sistémica continúa siendo el tratamiento de elección para la tromboembolia venosa, sin embargo, los filtros de vena cava son considerados como opción terapéutica importante en ciertos casos de pacientes que presenten alguna contraindicación.

Dentro de las complicaciones más frecuentes posteriores a su colocación se encuentran:

- La inclinación del filtro, la cual se define como una angulación del filtro superior a 15 grados desde el eje largo de la vena cava. Esto se puede ver con todos los filtros excepto con el filtro Bird's nest (Cook Medical, Bloomington, IN, EE.UU.) y está dado por la configuración propia de cada dispositivo ${ }^{1}$. Se documenta que la inclinación es la causa más común de falla en la recuperación de los filtros ${ }^{2}$. Rogers et al. encontraron que los filtros inclinados a más de 14 grados se asociaban con un aumento de la embolia pulmonar (EP), por lo que recomendaron la adición de un segundo filtro para una profilaxis adecuada, sin embargo en la actualidad esta recomendación no está vigente ${ }^{3}$. Los estudios sobre los filtros Greenfield (Boston Scientific, Natick, MA, EE.UU.) mostraron una incidencia de inclinación en el $5 \%$ de la colocación de filtros y una tendencia hacia un aumento de la trombosis de la vena cava inferior ( $\mathrm{VCl}$ ) y de EP en filtros inclinados en comparación con los no inclinados.

- Adicionalmente, la migración del filtro ocurre al documentar un movimiento superior o inferior de $2 \mathrm{~cm}$ o más desde la ubicación de la colocación inicial. Un filtro de tamaño insuficiente para la vena cava puede provocar migración. La mayoría de los filtros comercialmente aprobados actualmente para vena cava son de $28 \mathrm{~mm}$ o menos de diámetro, con la excepción del filtro Bird's nest, que se puede colocar en una vena cava de hasta $40 \mathrm{~mm}$ de diámetro ${ }^{4,5}$. Otros contribuyentes a la migración son la colocación de dispositivos intravasculares centrales, ya que pueden desalojar el filtro. Esto se puede prevenir con el uso de técnicas fluoroscópicas al colocar una vía central en este grupo de pacientes.

- Otra de las complicaciones es la apertura incompleta del filtro, que puede deberse a un defecto en el filtro, un error del operador $o$ un trombo no identificado en la $\mathrm{VCl}$, lo que conduce a una configuración anormal y asimétrica del filtro después de la implementación. Las tasas de incidencia oscilan entre el $0.7 \mathrm{y}$ el $13.9 \%^{2}$.
- La fractura del filtro ocurre cuando hay una falla estructural del dispositivo, provocando su ruptura y la potencial embolización del fragmento. A menudo, esta es una complicación tardía de los filtros, que se observa con mayor frecuencia después de que el filtro ha estado colocado durante más de un año ${ }^{5}$. Por lo que el aumento del tiempo implantado puede provocar una fatiga estructural del filtro. La incidencia global es del 1-2\% y se informó con mayor frecuencia en el filtro Greenfield 6 .

- La perforación del filtro se considera como una complicación grave, que ocurre cuando un componente del filtro penetra $>3 \mathrm{~mm}$ de la pared de la vena cava $y$ entra en el espacio pericaval y/o las estructuras adyacentes ${ }^{7}$. La perforación puede ocurrir inmediatamente al desplegar un filtro o como consecuencia tardía. Se cree que el movimiento de la $\mathrm{VCl}$ con pulsaciones aórticas y la respiración son los posibles mecanismos de esta última presentación tardía. Últimamente se han agregado ganchos a los filtros para disminuir la migración, afectando en el aumento de la incidencia de la perforación ${ }^{6}$. En general, el 20\% de las complicaciones en la base de datos MAUDE (Manufacturer And User Facility Device), que se encarga de recabar los informes y eventos adversos relacionados con los productos sanitarios, se explican por perforaciones de la $\mathrm{VCl}^{8}$. La incidencia de la perforación varía ampliamente según el tipo y el diseño del filtro. Los filtros Greenfield, Bird's nest y Simon-ninitol (CR Bard, Covington, GA, EE.UU.) suelen tener la mayor incidencia de perforación a nivel de la aorta y vena cava ${ }^{4,9}$. La presencia de esta complicación demanda la utilización de técnicas avanzadas endovasculares para su retiro o del retiro quirúrgico del filtro dependiendo del grado de perforación.

- Finalmente, la trombosis de la cava se ha citado por tener una incidencia cercana al $20 \%$ con filtros contemporáneos reportados en el año 2006, pero las tasas publicadas varían ampliamente entre el 2 y el $30 \%{ }^{10}$. La variabilidad probablemente se deba a las prácticas de detección de pacientes asintomáticos, ya que es diez veces más común que la trombosis de la cava sintomática ${ }^{11}$. Los síntomas incluyen dolor y edema de ambas extremidades inferiores, así como insuficiencia renal si el trombo se extiende hasta la $\mathrm{VCl}$ suprarrenal. Un filtro de $\mathrm{VCl}(\mathrm{FVCl})$ trombosado puede aumentar el riesgo de EP, ya que el trombo puede extenderse por encima del filtro $y$ luego la embolia conduce a EP recurrente en el contexto de un $\mathrm{FVCl}^{10}$. 
En el estudio PREPIC (Prevention du Risque d'Embolie Pulmonaire par Interruption Cave) se observó trombosis sintomática de la $\mathrm{VCl}$ en el $13 \%$ de los receptores del filtro después de ocho años de seguimiento $^{12}$. La incidencia de trombos de $\mathrm{VCl}$ varía aun más según el tipo de filtro, ya que se han informado tasas significativamente más altas de trombos de $\mathrm{VCl}$ para el filtro TrapEase (Cordis Corporation, Bridgewater, NJ) en comparación con otros filtros permanentes ${ }^{10,11}$. Un estudio observacional prospectivo de pacientes con filtros VenaTech (B. Braun/VenaTech, Evanston, IL) demostró trombosis de la $\mathrm{VCl}$ en el $33 \%$ de los pacientes después de nueve años de seguimiento ${ }^{12}$.

Debido a la diversidad y gravedad de las complicaciones asociadas al $\mathrm{FVCl}$ exponemos a continuación un caso clínico ilustrativo.

\section{Caso clínico}

Se trata de una paciente de sexo femenino de 43 años, con antecedente de síndrome de anticuerpos antifosfolípidos diagnosticado en 2009 al haber presentado además del perfil inmunológico compatible reportado 05/10/2009 anti-beta 2 glucoproteína (IgG) 4.4, anti-beta 2 glucoproteína (IgM) 0.5, anticardiolipina (IgM) 0.6, anticuerpos anti-SSB 3.3, cuenta con un óbito de ocho meses y un aborto en primer trimestre.

Se agrega trombosis venosa profunda (TVP) en miembro pélvico izquierdo en ese año, iniciando tratamiento con acenocumarina ( $2 \mathrm{mg}$ cada 24 horas), aunque con dificultad para alcanzar niveles terapéuticos de INR (International Normalized Ratio). Consecuentemente presenta tromboembolia pulmonar (TEP). En el abordaje diagnóstico se detecta además hipertensión pulmonar y se decide colocación de filtro de Greenfield en $\mathrm{VCl}$ asociado al tratamiento con acenocumarina. Al momento de la colocación del filtro presentó una complicación del acceso que fue resuelta con disección yugular cervical derecha.

Desde el año 2011 cambia su tratamiento a dosis profiláctica de enoxaparina (60 mg cada 12 horas) y aspirina (100 mg cada 24 horas), ya que la paciente deseaba embarazarse. En el 2015 suspende por completo la anticoagulación y continúa únicamente con aspirina (100 mg cada 24 horas). Presenta TEP segmentaria de bajo riesgo en rama segmentaria basal anterior del lóbulo inferior derecho sin zona de infarto asociada y TVP de miembro pélvico derecho con edema que incapacita la movilidad adecuada de la extremidad e índice tobillo-brazo preservado (tibial anterior derecho 1.1, tibial posterior derecho 1). Por

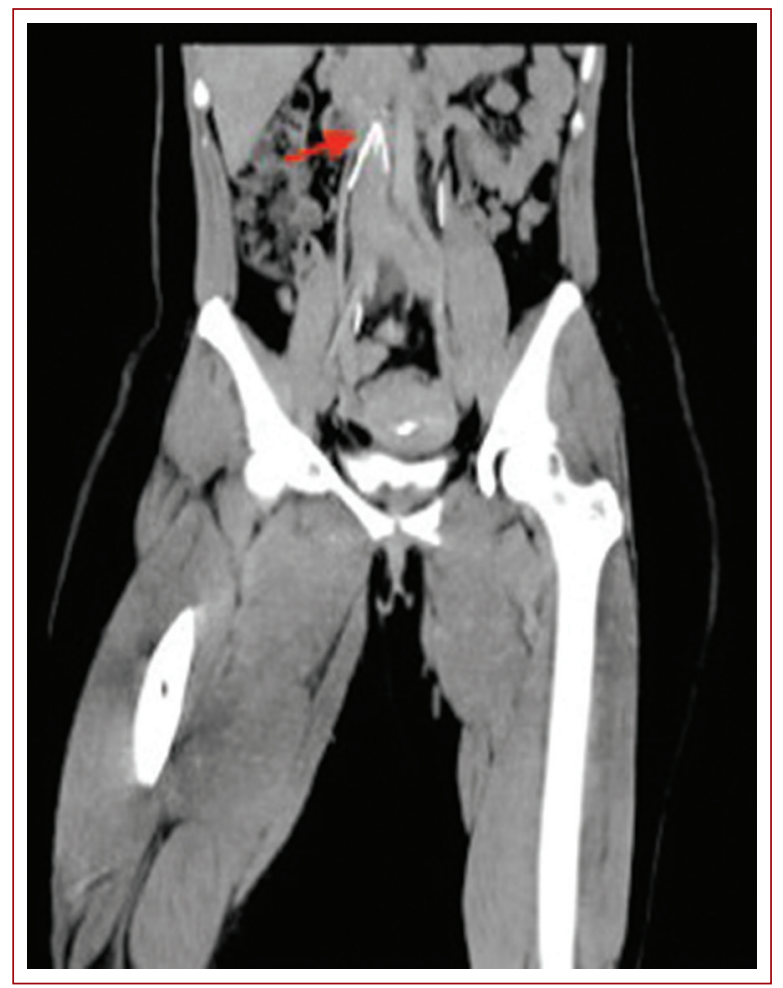

Figura 1. Corte coronal de tomografía de abdomen que muestra defecto de llenado posterior al filtro de vena cava inferior secundario a trombosis a este nivel.

angiotomografía se observa un defecto de llenado persistente posterior al $\mathrm{FVCl}$ secundario a trombosis a este nivel e infragenicular derecho (Fig. 1).

Se realiza ajuste de anticoagulación y utilización de medias de compresión elástica de $30-40 \mathrm{mmHg}$. Dos semanas después reingresa con nuevo episodio de trombosis venosa iliofemoral, ahora izquierda, sobre anticoagulación subóptima. En esta ocasión presenta edema de mayor importancia que el de la extremidad contralateral sin flegmasía. Debido a los eventos de repetición de TVP, el EP a pesar de la presencia del filtro, la ausencia de contraindicación para anticoagulación oral y hallazgo de oclusión total del $\mathrm{FVCl}$ con trombosis de la vena cava con perforación de su pared hacia la aorta (Fig. 2) se decide la extracción abierta del filtro de vena cava.

El retiro del filtro se realizó mediante un abordaje transperitoneal, con movilización visceral medial y exposición de la vena cava desde su segmento infrahepático hasta las venas ilíacas comunes. Se demuestra la perforación de la cava adyacentes a zonas de abundante fibrosis pericaval y perforación de la adventicia aórtica en uno de sus ganchos (Fig. 3). Una vez realizado el control vascular, es únicamente posible el 


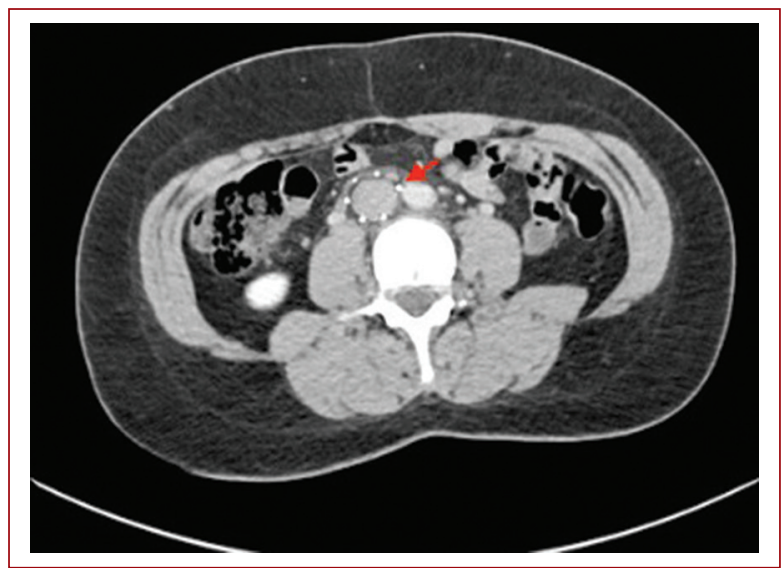

Figura 2. Corte transversal en tomografía de abdomen que muestra perforación de la pared de la vena cava inferior por los ganchos del filtro.

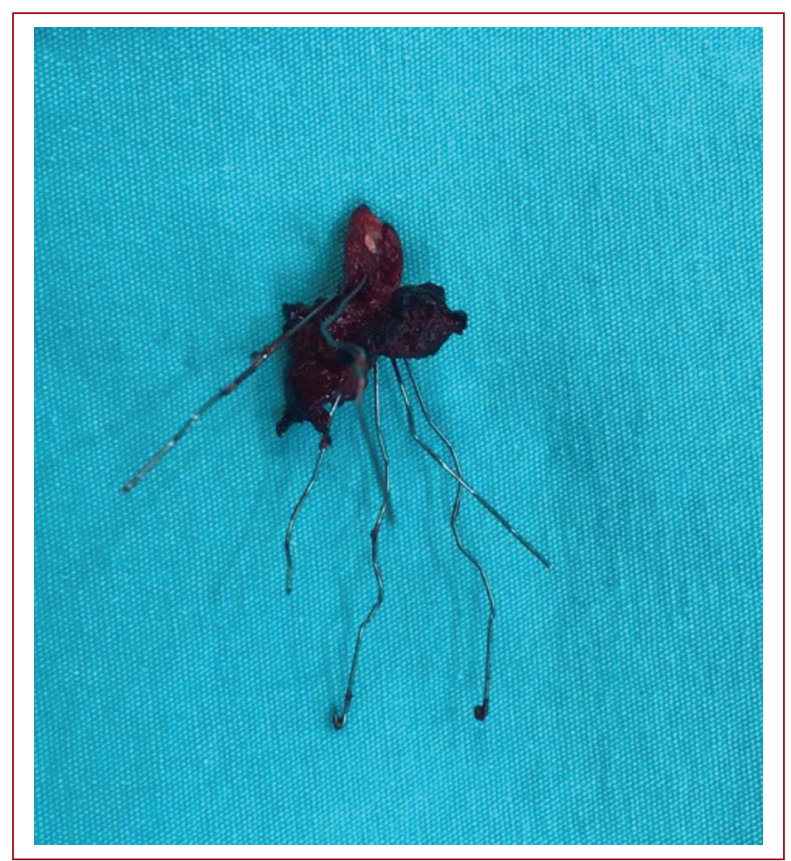

Figura 3. Filtro de vena cava extraído. Los ganchos perforaban la pared de la vena cava inferior y de la aorta.

retiro del filtro una vez fragmentados cada uno de los ganchos y realizado el retiro independiente de estos. Además, debido a la calidad aguda $<2$ semanas de evolución) del trombo se realizó trombectomía cava y de ilíaca izquierda seguida de trombectomía venosa del miembro pélvico izquierdo (Fig. 4). Debido a la cronicidad de la trombosis derecha, se continuó con manejo conservador. Como hallazgo interesante se

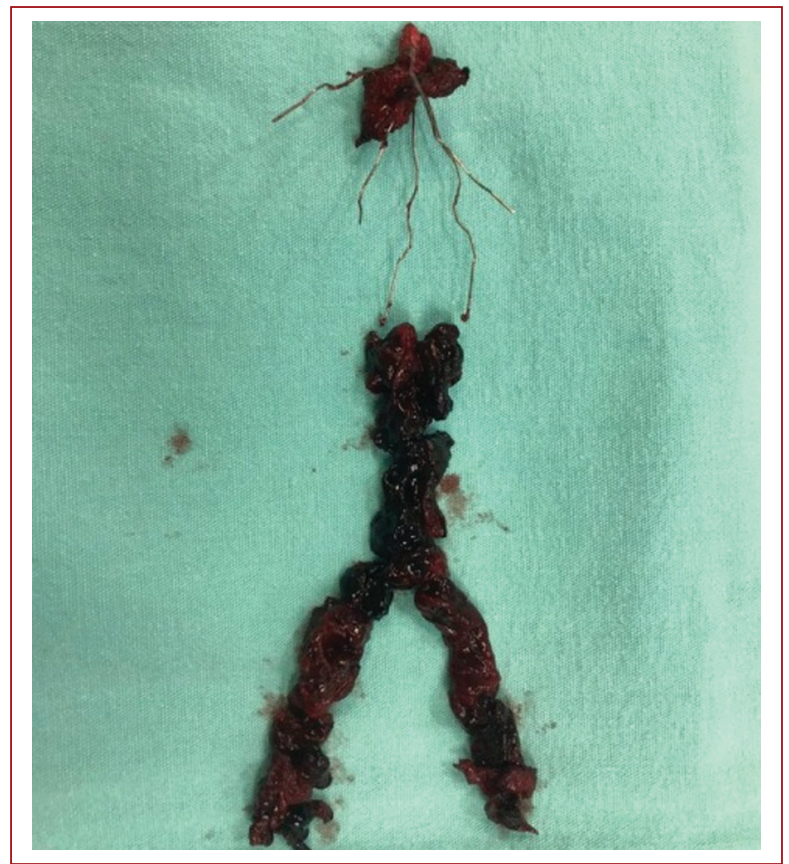

Figura 4. Se extraen trombos de la vena cava inferior y de la vena ilíaca común izquierda.

encontró una vena ovárica derecha hipertrófica que desembocaba por arriba del FVCI.

A partir de esta intervención, durante su seguimiento la paciente persiste sin eventos trombóticos de repetición, con anticoagulación en dosis óptima y resolución de síntomas en extremidades inferiores. Asimismo, permanece sin síndrome posflebítico de acuerdo con el score de Villalta (3 puntos).

\section{Discusión}

El pilar del tratamiento de los pacientes con enfermedad tromboembólica venosa es la anticoagulación. Los pacientes anticoagulados tienen tasas bajas de tromboembolia venosa recurrente y los pacientes con TVP tienen tasas bajas de desarrollo posterior de EP. Antes de la práctica actual de la anticoagulación, la profilaxis primaria de enfermedad pulmonar a menudo consistía en ligadura quirúrgica o interrupción de la $\mathrm{VCl}$ como medio para interrumpir la enfermedad pulmonar. Estos procedimientos quirúrgicos sentaron las bases para los filtros en la $\mathrm{VCl}$ que se utilizan en la actualidad.

La primera descripción de un trombo se realizó en la época antes de Cristo. Hipócrates en el 460 a.C. y Aristóteles en el 322 a.C. postulaban que esta transformación del estado líquido al sólido se producía 


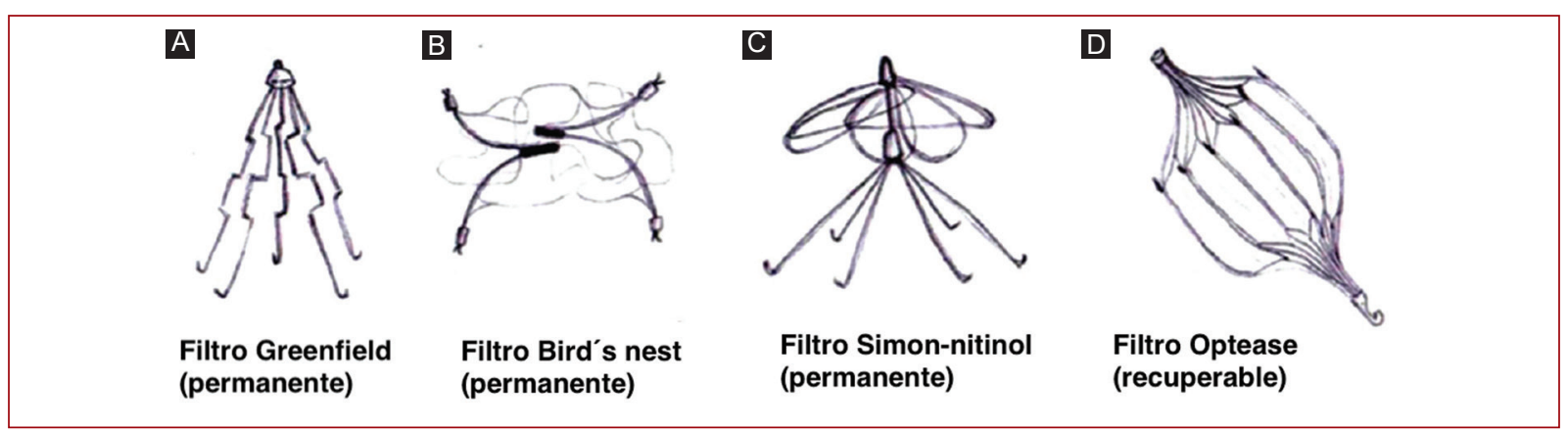

Figura 5. Se muestran ilustraciones sobre los distintos tipos de filtros de vena cava. Filtro Greenfield (A), filtro Bird's nest (B) y filtro Simon-nitinol (C), los cuales son permanentes, y filtro Optease (D), el único filtro al momento recuperable.

cuando la sangre que se encontraba caliente dentro del cuerpo era expulsada al frío que había fuera de este $^{13}$.

El primer caso bien documentado de una trombosis corresponde a Guillaume de Saint Pathus, que describió el caso de una joven de 20 años que presentó edema en el tobillo derecho que fue progresando paulatinamente hasta el muslo ${ }^{14}$.

Consecutivamente se fueron estudiando tanto la TVP como la TEP, la cual se producía a consecuencia de la primera. Por lo que se realizaron los primeros avances en el tratamiento efectivo sobre la TVP para evitar que el coágulo formado en esta entidad pueda migrar hacia el corazón y posteriormente llegar a localizarse en el pulmón. A mediados de los 40, Oscher, De Bakey y O'Neil propusieron ligar la VCI para prevenir la TEP originada en los miembros inferiores y en la pelvis. Se reducía la recurrencia de TEP, pero requería de cirugía mayor, conllevando una mortalidad del $12 \%{ }^{15}$.

La idea inicial de utilización de filtros de vena cava surgió en 1967 en Búfalo, Nueva York, en un trabajo de Peter Eichlter y Worthington G. Shenk titulado Un nuevo enfoque experimental en la profilaxis de la embolia pulmonar. El primer dispositivo fue implantado en ese mismo año. Se le llamó «filtro de Mobin-Uddin» y fue liberado vía transyugular con un sistema de liberación de 27 Fr. La membrana de silicona fenestrada de la parte superior de la sombrilla fue diseñada con la intención de ocluir casi totalmente la VCI. Poco tiempo después se introdujo el filtro de Kimray-Greenfield en la práctica clínica y rápidamente se convirtió en el de uso preferido, ya que no producía tanta limitación del flujo y se redujo la incidencia de oclusión de $\mathrm{VCl}$, que variaba entre el 2 y el $28 \%$, incluso por debajo de los filtros Bird's nest, Simon-nitinol y Optease ${ }^{15}$ (Fig. 5).

El filtro Greenfield de titanio (TGF) (Boston Scientific, Natick, MA, EE.UU.), mismo que fue colocado en esta paciente, utilizaba un sistema portador $12 \mathrm{Fr}$ en lugar del sistema $24 \mathrm{Fr}$ utilizado para la inserción operativa del filtro Greenfield original de acero inoxidable (SGF). El TGF ha demostrado ser comparable al SGF a largo plazo en lo que respecta a la permeabilidad de la vena cava $(99 \%)$ y la protección contra EP $(97 \%)$. Sin embargo, debido a las limitaciones de diseño, la guía se eliminó durante la inserción, y esto pudo haber sido la causa del $10 \%$ de incidencia de asimetría de las extremidades que se observó.

Los ganchos se acortaron de 0.05 a 0.035 pulgadas y se alteró la dirección vertical en dos ramas para acomodar la guía. Aunque estos cambios de diseño fueron sutiles, existía la posibilidad de efectos adversos ${ }^{16}$. En este caso, el filtro se encontraba completamente endotelizado, por lo que producía una obstrucción total del flujo de retorno. Estos hallazgos se corroboraron debido a la presencia de una pared fina y delgada por arriba del filtro contrastante con la pared esclerótica por debajo del filtro, resultado de la inflamación derivada de los eventos repetidos de trombosis de la $\mathrm{VCl}$. Mismas trombosis que se asociaron al reciente evento de EP registrado o más probablemente debidos a una trombosis ocurrida por arriba del FVCl.

Las indicaciones actuales para los filtros de $\mathrm{VCl}$ varían de acuerdo con las distintas guías presentes como, por ejemplo:

- Guías ESC (European Society of Cardiology) 2019: indicaciones potenciales son la TVP, así como la contraindicación absoluta para el tratamiento con anticoagulante, la TEP recurrente y como profilaxis 
Tabla 1. Indicaciones para colocación de filtro de vena cava inferior. Comparación de las guías de práctica clínica

\begin{tabular}{|c|c|c|c|c|c|}
\hline Posible indicación & ACCP & SIR & AHA & BCSH & ESC/ERA \\
\hline $\begin{array}{l}\text { Trombosis venosa profunda } \\
\text { proximal o embolia pulmonar y } \\
\text { contraindicación para } \\
\text { anticoagulación }\end{array}$ & $\begin{array}{l}\text { Recomendado } \\
\text { (grado 1C) }\end{array}$ & $\begin{array}{l}\text { Recomendado } \\
\text { (indicación absoluta) }\end{array}$ & $\begin{array}{l}\text { Recomendado } \\
\text { (clase I) }\end{array}$ & Recomendado & $\begin{array}{l}\text { Recomendado } \\
\text { (clase Ila) }\end{array}$ \\
\hline Falla a la anticoagulación & - & - & $\begin{array}{l}\text { Sugerido } \\
\text { (clase Ila) }\end{array}$ & Considerar & $\begin{array}{l}\text { Recomendado } \\
\text { (clase Ila) }\end{array}$ \\
\hline $\begin{array}{l}\text { Embolia pulmonar masiva con } \\
\text { trombosis venosa residual }\end{array}$ & - & - & - & - & - \\
\hline $\begin{array}{l}\text { Trombosis venosa libre proximal } \\
\text { o iliocava }\end{array}$ & - & $\begin{array}{l}\text { Recomendado } \\
\text { (indicación relativa) }\end{array}$ & $\begin{array}{l}\text { No recomendado } \\
\text { (clase III) }\end{array}$ & $\begin{array}{l}\text { No } \\
\text { recomendado }\end{array}$ & $\begin{array}{l}\text { No } \\
\text { recomendado }\end{array}$ \\
\hline $\begin{array}{l}\text { Enfermedad cardiopulmonar } \\
\text { severa y trombosis venosa } \\
\text { profunda }\end{array}$ & - & $\begin{array}{l}\text { Recomendado } \\
\text { (indicación relativa) }\end{array}$ & - & - & - \\
\hline $\begin{array}{l}\text { Previo a tromboendarterectomía } \\
\text { para hipertensión pulmonar } \\
\text { tromboembólica crónica }\end{array}$ & $\begin{array}{l}\text { Sugerido } \\
\text { (grado } 2 \mathrm{C} \text { ) }\end{array}$ & $\begin{array}{l}\text { Recomendado } \\
\text { (indicación relativa) }\end{array}$ & - & - & $\begin{array}{l}\text { No } \\
\text { recomendado }\end{array}$ \\
\hline Previo a trombosis & - & $\begin{array}{l}\text { Recomendado } \\
\text { (indicación relativa) }\end{array}$ & - & $\begin{array}{l}\text { No } \\
\text { recomendado }\end{array}$ & $\begin{array}{l}\text { No } \\
\text { recomendado }\end{array}$ \\
\hline
\end{tabular}

ACCP: American College of Chest Physicians; SIR: Society of Interventional Radiology, AHA: American Heart Association; BCSH: British Committee for Standards in Hematology, ESC: European Society of Cardiology, ERA: European Respiratory Society.

primaria en pacientes con TVP de alto riesgo, además de presencia de trombos de libre flotación ${ }^{17}$.

- Guías CHEST (American College of Chest Physicians) del 2016: a) pacientes con tromboembolia venosa aguda y contraindicación a la anticoagulación, recomendar el uso de un filtro $\mathrm{VCl}$; b) en pacientes con EP de alto riesgo/masiva, considere el $\mathrm{FVCI}$ además de la anticoagulación, y c) en pacientes con tromboembolia venosa recurrente a pesar de una anticoagulación adecuada, el filtro VCl es una opción de último recurso ${ }^{18}$.

- Guías de la AHA (American Heart Association) del 2016 y guías ASH (American Society of Hematology) 2020: a) los pacientes adultos con cualquier EP, aguda confirmada (o TVP proximal) con contraindicaciones para la anticoagulación o con una complicación hemorrágica activa deben recibir un filtro $\mathrm{VCl}$; b) para pacientes con EP aguda recurrente a pesar de la anticoagulación terapéutica, es razonable colocar un filtro $\mathrm{VCl}$, y c) se puede considerar la colocación de un FVCI para pacientes con EP aguda y reserva cardiopulmonar muy pobre, incluidos aquellos con EP masiva/de alto riesgo ${ }^{19,20}$ (Tabla 1).

Analizando el caso presentado anteriormente, la paciente ya contaba con dos eventos previos de TVP y de EP, sobre anticoagulación subóptima. Los eventos iniciales de TEP ocurrieron durante su vigilancia en otra institución, pero fue considerada para colocación de filtro debido a esos eventos tromboembólicos y su estado protrombótico de base. Sin embargo, hay que considerar que las indicaciones que regían en esa época la colocación de un filtro difieren de las actuales. El desconocimiento de las complicaciones a largo plazo de la utilización de filtros permanentes incitó su uso indiscriminado en algunos casos, mismos que bajo las normas actuales sería controvertido. Además, la indicación de colocación de filtro tuvo lugar hace 16 años debido a las irregularidades en su anticoagulación alrededor del embarazo, conocido factor protrombótico per se. Desde esa oportunidad no se le retiró debido a que inicialmente los filtros eran permanentes en su totalidad, pues se desconocían las consecuencias del uso de estos a largo plazo. Por ello, como se ha discutido, actualmente su uso, como recomendación clase I, se ha restringido a aquellos con trombosis venosa proximal y contraindicación para la anticoagulación, buscando siempre el retiro a corto plazo de estos, siguiendo la tendencia actual de colocación de filtros recuperables, así como continuar con anticoagulación una vez que se haya resuelto el motivo de la contraindicación. 
Precisamente debido a esta situación, en 2010 la FDA (Food and Drug Administration) emitió un comunicado de seguridad instando a los médicos a que colocaran $\mathrm{FVCl}$ recuperables. La comunicación de seguridad fue en respuesta a más de 900 informes de eventos adversos relacionados con filtros VCl. Se enfatizó en las complicaciones como la fractura del filtro, la embolización, la migración, la penetración de la pared de la cava y trombosis como las más preocupantes.

Cuando se realiza el retiro en sala de hemodinamia, las complicaciones del procedimiento durante la colocación del $\mathrm{FVCl}$ son poco frecuentes y, por lo general, tienen poca importancia clínica. Un estudio que detalla la experiencia en un solo centro con filtros $\mathrm{VCl}$ en casi tres décadas informó una tasa de complicaciones mayores del $0.3 \%$. En raras ocasiones, puede haber un problema con el despliegue del filtro, ocasionando la recuperación y su reemplazo inmediato ${ }^{21}$.

La penetración de la pared de la vena cava que ocurre en este caso implica que las puntas del filtro sobresalgan más allá de los límites de la pared con posibilidad de perforación hacia los órganos adyacentes. En el caso de la conformación de los ganchos del filtro de Greenfield, su penetración imposibilita la extracción endovascular pues se encuentran en forma de $U$ y por lo tanto atraparían la pared venosa al momento de la recuperación del dispositivo y condicionarían un desgarro grave de la cava y de la adventicia aórtica de forma simultánea. Existen múltiples informes que describen esta complicación. En general, se informó penetración de la pared de la vena cava en el $19 \%$ de los pacientes $(1,699$ de 9,002$)$ y el $19 \%$ de esas penetraciones (322 de 1,699) mostró evidencia de afectación de órganos/estructuras sobre todo vasculares como la aorta. El más conocido fue una revisión sistemática del 2015 publicada en Circulation, que incluía estudios entre 1970 y 2014 relacionados con $\mathrm{FVCl}$; un total de 88 estudios clínicos y 112 informes de casos calificados para el análisis, estos estudios incluyeron 9,002 pacientes y 15 tipos de $\mathrm{FVCl}$.

Entre los pacientes con penetración, el $8 \%$ era sintomático, el $45 \%$ estaba asintomático y el $47 \%$ tenía sintomatología desconocida. El síntoma informado con más frecuencia fue el dolor $(77 \%, 108$ de 140). Se informaron complicaciones importantes en 83 pacientes $(5 \%)^{22,23}$, dadas por perforación del filtro, inclinación, migración y oclusión del filtro, principalmente. Nuestro caso se consideraría como sintomático debido a la presencia de TVP y edema asociado al enlentecimiento del retorno venoso por la obstrucción total del filtro además de la penetración de las paredes vasculares aorto-cavas.

Cabe recalcar que el último metaanálisis publicado en el 2018 en el Journal of the American College of Cardiology, referente a la utilización del $\mathrm{FVCl}$ para prevenir $\mathrm{EP}$, los pacientes que recibieron $\mathrm{FVCl}$ tuvieron un riesgo menor de EP posterior (odds ratio [OR]: 0.50), mayor riesgo de TVP (OR: 1.70), mortalidad relacionada con EP no significativamente menor (OR: 0.51) y ningún cambio en la mortalidad por todas las causas (OR: 0.91). De ahí el cambio de paradigma en su utilización.

De forma complementaria y como medida de prevención de síndrome posflebítico, se deben de agregar medidas de contención elástica, ya sea por medio de un vendaje elástico o por medias de compresión elástica alta o adecuada al grado de insuficiencia arterial de cada caso. Adicionalmente esta maniobra permite que se reduzca el volumen de sangre que mantiene el paciente, drenándola al sistema venoso periférico y aliviando la tensión, el edema y el malestar subjetivo del paciente ${ }^{24}$.

Finalmente, el manejo con trombectomía ofrece mejoría considerable de los síntomas y preserva la función valvular, ya que se realizó a favor de la dirección valvular al contar con exposición femoral y cava. Esto mejora el pronóstico en cuanto a posibles secuelas posflebíticas a largo plazo en la pierna izquierda. En el lado derecho, la hipertrofia de la vena ovárica demuestra una compensación adecuada de la obstrucción crónica por estas colaterales naturales y por lo tanto se puede esperar una recuperación aceptable del evento agudo. Actualmente la paciente se encuentra con adecuada permeabilidad venosa, buen estado general y niega datos de claudicación.

\section{Conclusión}

El uso de filtros de vena cava debe limitarse a pacientes con trombosis venosa aguda que presenten contraindicaciones para recibir anticoagulación. En caso de indicarse, es necesario realizar una vigilancia estrecha y el retiro correspondiente, al resolver la causa de la contraindicación para anticoagulación con el fin de prevenir complicaciones.

\section{Financiamiento}

La presente investigación no ha recibido ninguna beca específica de agencias de los sectores público, comercial o con ánimo de lucro. 


\section{Conflicto de intereses}

Los autores declaran no tener conflicto de intereses.

\section{Responsabilidades éticas}

Protección de personas y animales. Los autores declaran que para esta investigación no se han realizado experimentos en seres humanos ni en animales.

Confidencialidad de los datos. Los autores declaran que han seguido los protocolos de su centro de trabajo sobre la publicación de datos de pacientes.

Derecho a la privacidad y consentimiento informado. Los autores declaran se ha obtenido el consentimiento informado del paciente referido en el artículo.

\section{Bibliografía}

1. Neeman Z, Auerbach A, Wood BJ. Metastatic involvement of a retrieved inferior vena cava filter. J Vasc Interv Radiol. 2003;14(12):1585.

2. Joels CS, Sing RF, Heniford BT. Complications of inferior vena cava filters. Am Surg. 2003;69(8):654-9.

3. Rogers FB, Strindberg G, Shackford SR, et al. Five-year follow-up of prophylactic vena cava filters in high-risk trauma patients. Arc Surg. 1998;133:406-11.

4. Martin MJ, Blair KS, Curry TK, Singh N. Vena cava filters: Current concepts and controversies for the surgeon. Curr Probl Surg. 2010;47:524-618.

5. Kalva SP, Wicky S, Waltman AC, Athanasoulis CA. TrapEase vena cava filter: experience in 751 patients. J Endovasc Ther. 2006;13(3):365-72.

6. Carman TL, Alahmad A. Update on vena cava filters. Curr Treat Options Cardiovasc Med. 2008;10(2):101-11.

7. McLoney ED, Krishnasamy VP, Castle JC, Yang X, Guy G. Complications of Celect, Günther tulip, and Greenfield inferior vena cava filters on CT follow-up: a single-institution experience. J Vasc Interv Radiol. 2013;24(11):1723-9.

8. Angel LF, Tapson V, Galgon RE, Restrepo MI, Kaufman J. Systematic review of the use of retrievable inferior vena cava filters. J Vasc Interv Radiol. 2011;22(11):1522-1530.e3.

9. Ray Jr C, Kaufman J. Complications of inferior vena cava filters. Abdom Imaging. 1996;21:368-74.
10. Milovanovic L, Kennedy SA, Midia M. Procedural and indwelling complications with inferior vena cava filters: frequency, etiology, and management. Semin Intervent Radiol. 2015;32(1):34-41.

11. Ahmad I, Yeddula K, Wicky, S. Clinical sequelae of thrombus in an inferior vena cava filter. Cardiovasc Intervent Radiol. 2010;33:285-9.

12. Decousus H, Leizorovicz A, Parent F, Tardy B, Girard P, Laporte S, et al. A clinical trial of vena caval filters in the prevention du Ris-que d'Embolie Pulmonaire par Interruption Cave Study Group. N Engl J Med. 1998;338:409-15.

13. Shapiro SS. Treating thrombosis in the $21^{\text {st }}$ century. $\mathrm{N}$ Engl $\mathrm{J}$ Med. 2003;349(18):17624

14. Mannucci PM. Venous thrombosis: the history of knowledge. Pathophysiol Haemost Thromb. 2002;32(5-6):209-12.

15. Harris L, Blochle R. Haimovici's vascular surgery. $12^{\text {th }}$ ed. Wiley-Blackwell; 2012.

16. Greenfield LJ, Proctor MC, Cho KJ, Cutler BS, Ferris EJ, McFarland D, et al. Extended evaluation of the titanium Greenfield vena caval filter. J Vasc Surg. 1994;20(3):458-64; discussion 464-5.

17. Konstantinides SV, Meyer G, Becattini C, Bueno H, Geersing G-J, Harjola V-P, et al.; Grupo de Trabajo de la Sociedad Europea de Cardiología (ESC) para el diagnóstico y tratamiento de la tromboembolia pulmonar aguda. Guía ESC 2019 para el diagnóstico y tratamiento de la embolia pulmonar. Rev Esp Cardiol. 2020;73(6):497.e1-497.e58

18. Kearon C, Akl EA, Ornelas J, Blaivas A, Jimenez D, Bounameaux H, et al. Antithrombotic therapy for VTE disease: CHEST Guideline and Expert Panel Report. Chest. 2016;149(2):315-52.

19. Jaff MR, McMurtry MS, Archer SL, Cushman M, Goldenberg N, Goldhaber SZ; American Heart Association Council on Cardiopulmonary, Critical Care, Perioperative and Resuscitation; American Heart Association Council on Peripheral Vascular Disease; American Heart Association Council on Arteriosclerosis, Thrombosis and Vascular Biology. Management of massive and submassive pulmonary embolism, iliofemoral deep vein thrombosis, and chronic thromboembolic pulmonary hypertension: a scientific statement from the American Heart Association. Circulation. 2011;123(16):1788-830.

20. Ortel TL, Neumann I, Ageno W, Beyth R, Clark NP, Cuker A, et al. American Society of Hematology 2020 guidelines for management of venous thromboembolism: treatment of deep vein thrombosis and pulmonary embolism. Blood Adv. 2020;4:4693-738.

21. Athanasoulis CA, Kaufman JA, Halpern EF, Waltman AC, Geller SC, Fan CM. Inferior vena caval filters: review of a 26 -year single-center clinical experience. Radiology. 2000;216(1):54-66.

22. Jia Z, Wu A, Tam M, Spain J, McKinney JM, Wang W. Caval penetration by inferior vena cava filters: A systematic literature review of clinical significance and management. Circulation. 2015;132(10):944-52.

23. Bos A, van Ha T, van Beek D, Ginsburg M, Zangan S, Navuluri R, et al. Strut penetration: local complications, breakthrough pulmonary embolism, and retrieval failure in patients with Celect vena cava filters. J Vasc Interv Radiol. 2015;26(1):101-6.

24. Viver Manresa E. Patología vascular. 1.ํㅡㄹ edición. Barcelona, España: Edikamed; 1993. 